\title{
CARRY TRADE: THE GAINS OF DIVERSIFICATION
}

\section{Craig Burnside}

Duke University

\author{
Martin Eichenbaum \\ Northwestern University
}

\author{
Sergio Rebelo \\ Northwestern University
}

\begin{abstract}
Market participants routinely take advantage of the failure of uncovered interest rate parity to speculate in currency markets. Perhaps the most widely used currency speculation strategy is the carry trade. In this article we take the perspective of an individual currency trader and document the gains to diversifying the carry trade across different currencies. We show that these gains are large. Diversification boosts the typical Sharpe ratio by over $50 \%$.
\end{abstract}

\section{Introduction}

It is well-known that uncovered interest rate parity (UIP) does not hold. ${ }^{1}$ UIP implies that high interest rate currencies should depreciate vis-à-vis low interest rate currencies. In fact, on average, they appreciate. Market participants routinely take advantage of the failure of UIP to speculate in currency markets. Perhaps the most widely used currency speculation strategy is the carry trade. This strategy involves selling forward currencies that are at a forward premium (i.e., the forward rate exceeds the spot exchange rate) and buying forward currencies that are at a forward discount. Transactions costs aside, this strategy is equivalent to borrowing low interest rate currencies, lending high interest rate currencies, and not hedging exchange rate risk.

In practice one can apply the carry trade to individual currencies or to portfolios of currencies. Burnside et al. (2006) consider the payoff to an equally weighted portfolio of carry-trade strategies. This portfolio of strategies is constructed as follows. In every period the investor places an identical dollar bet in

Acknowledgments: Burnside is grateful to the National Science Foundation for financial support (SES-0516697). The authors are members of the NBER.

E-mail addresses: Burnside: burnside@econ.duke.edu; Eichenbaum: eich@northwestern.edu; Rebelo: s-rebelo@ kellogg.northwestern.edu

1. See Bilson (1981) and Fama (1984) for early empirical work documenting the failure of UIP. See Hodrick (1987) and Engel (1996) for surveys of the literature. 
all the currencies, selling forward those that are at a premium and buying forward those that are at a discount. Burnside et al. show that the Sharpe ratio generated by this strategy is positive and statistically different from zero. Perhaps more importantly, the payoffs of the strategy are uncorrelated with traditional risks factors. So the payoff to the carry trade cannot be interpreted as compensation for bearing risk.

In this article we take the perspective of an individual currency trader and document the gains to diversifying the carry trade across different currencies. We show that these gains are large. Diversification boosts the typical Sharpe ratio by over 50\%. This large rise in the Sharpe ratio reflects the imperfect correlation between carry-trade payoffs for different currencies. Of course, it is possible that a large low-probability shock could drive all payoffs down together and negate the gains from diversification. But, at least in our sample, such a shock did not occur. In this sense our results give lie to traders' aphorism that "when markets go down, the only thing that goes up is correlation."

Our article is organized as follows. In Section 2 we describe the data used in our analysis. In Section 3 we discuss three carry-trade strategies and present our results. Section 4 contains concluding remarks.

\section{Data}

Our data are from Datastream and cover the period from January 1976 to June 2007. We incorporate currencies into our analysis as data on them become available. Each exchange rate is quoted as foreign currency units per U.S. dollar. We construct a monthly data set by sampling daily data on the last day of each month. Our data include both bid and ask exchange rates. The ask (bid) exchange rate is the rate at which a participant in the interdealer market can buy (sell) U.S. dollars from a currency dealer.

There are 23 countries in our sample: Australia, Austria, Belgium, Canada, Denmark, Finland, France, Germany, Greece, Hong Kong, Ireland, Italy, Japan, Netherlands, New Zealand, Norway, Portugal, Singapore, South Africa, Spain, Sweden, Switzerland, and the U.K. Our sample also includes the Euro after 1999. To assess the robustness of our results we report results for both the full sample and the post-Euro period (January 1999 to June 2007).

\section{Three Carry-trade Strategies}

We consider a version of the carry-trade strategy that takes into account bidask spreads both in the design of the trading strategy and in the computation of payoffs. Let $S_{t}^{a}$ and $S_{t}^{b}$ denote the ask and bid spot exchange rates, respectively. Let $F_{t}^{a}$ and $F_{t}^{b}$ denote the ask and bid forward exchange rate, respectively, for 
forward contracts maturing at time $t+1$. All exchange rates are expressed as foreign currency units per U.S. dollar.

We sell $x_{t}$ dollars forward according to the rule:

$$
x_{t}= \begin{cases}+1 & \text { if } F_{t}^{b} / S_{t}^{a}>1, \\ -1 & \text { if } F_{t}^{a} / S_{t}^{b}<1, \\ 0 & \text { otherwise }\end{cases}
$$

The carry trade is a zero net investment strategy; in equation (1) we normalize the size of the bet to one. The realized payoffs are given by:

$$
z_{t+1}= \begin{cases}x_{t}\left(F_{t}^{b} / S_{t+1}^{a}-1\right) & \text { if } x_{t}>0 \\ x_{t}\left(F_{t}^{a} / S_{t+1}^{b}-1\right) & \text { if } x_{t}<0 \\ 0 & \text { if } x_{t}=0\end{cases}
$$

We consider three strategies. In the "equally weighted carry-trade" strategy the dollar position gives equal weight at each point in time to all the currencies for which we have data and for which $x_{t}$ is different from zero. We normalize the total size of the bet to one dollar. In the "currency-specific carry-trade" strategy we apply equation (1) to an individual currency, normalizing the size of the bet to one dollar. In the "high-low carry-trade" strategy we take a position only in the two currencies with the highest forward premium and discount. At each point in time we sell the U.S. dollar forward against the currency that has the highest value of $F_{t}^{b} / S_{t}^{a}$ and we buy the U.S. dollar forward against the currency that has the smallest value of $F_{t}^{a} / S_{t}^{b}{ }^{2}$ If $1 / S_{t}^{a}$ and $1 / S_{t}^{b}$ are martingales, this strategy corresponds to buying and selling the two forward contracts with the highest expected payoffs.

Table 1 reports the mean, standard deviation, and Sharpe ratio associated with the excess returns to our three strategies. Consider first the results for the equally weighted carry-trade strategy. The Sharpe ratio is positive and statistically different from zero for both samples. On an annualized basis the Sharpe ratio is 0.83 for the whole sample and 1.23 for the post-Euro sample. In contrast, the annualized median Sharpe ratio across currency-specific carry trades is 0.50 for the whole sample and 0.54 for the post-Euro sample. Finally, the annualized average Sharpe ratio for the high-low carry-trade strategy is 0.54 for the whole sample and 0.55 for the post-Euro sample. Clearly, there are large gains from diversifying the carry trade across different currencies.

Relative to the high-low carry trade, the rise in the Sharpe ratio associated with the equally weighted carry trade is mostly due to a $34 \%$ decline in volatility.

2. There are instances in our sample in which there are no currencies at a forward discount against the U.S. dollar. In these cases we double the size of the bet on the currency that has the highest forward premium. 
TABLE 1. Excess returns to the carry-trade strategies (statistics presented on an annualized basis).

\begin{tabular}{|c|c|c|c|c|c|c|}
\hline & \multicolumn{3}{|c|}{ 1976M1-2007M6 } & \multicolumn{3}{|c|}{ 1999M1-2007M6 } \\
\hline & Mean & $\begin{array}{c}\text { Standard } \\
\text { Deviation }\end{array}$ & $\begin{array}{c}\text { Sharpe } \\
\text { Ratio }\end{array}$ & Mean & $\begin{array}{c}\text { Standard } \\
\text { Deviation }\end{array}$ & $\begin{array}{c}\text { Sharpe } \\
\text { Ratio }\end{array}$ \\
\hline Australia & $\begin{array}{c}0.109 \\
(0.041)\end{array}$ & $\begin{array}{c}0.088 \\
(0.006)\end{array}$ & $\begin{array}{c}1.238 \\
(0.465)\end{array}$ & $\begin{array}{c}0.102 \\
(0.042)\end{array}$ & $\begin{array}{c}0.087 \\
(0.006)\end{array}$ & $\begin{array}{c}1.171 \\
(0.475)\end{array}$ \\
\hline Austria & $\begin{array}{c}0.042 \\
(0.030)\end{array}$ & $\begin{array}{c}0.114 \\
(0.006)\end{array}$ & $\begin{array}{c}0.367 \\
(0.267)\end{array}$ & & & \\
\hline Belgium & $\begin{array}{c}0.024 \\
(0.032)\end{array}$ & $\begin{array}{c}0.113 \\
(0.009)\end{array}$ & $\begin{array}{c}0.210 \\
(0.294)\end{array}$ & & & \\
\hline Canada & $\begin{array}{r}0.035 \\
(0.013)\end{array}$ & $\begin{array}{c}0.056 \\
(0.003)\end{array}$ & $\begin{array}{c}0.627 \\
(0.241)\end{array}$ & $\begin{array}{c}0.013 \\
(0.032)\end{array}$ & $\begin{array}{c}0.071 \\
(0.005)\end{array}$ & $\begin{array}{c}0.184 \\
(0.450)\end{array}$ \\
\hline Denmark & $\begin{array}{c}0.095 \\
(0.020)\end{array}$ & $\begin{array}{c}0.103 \\
(0.006)\end{array}$ & $\begin{array}{c}0.922 \\
(0.213)\end{array}$ & $\begin{array}{c}0.076 \\
(0.031)\end{array}$ & $\begin{array}{c}0.086 \\
(0.007)\end{array}$ & $\begin{array}{c}0.882 \\
(0.372)\end{array}$ \\
\hline Finland & $\begin{array}{c}0.025 \\
(0.060)\end{array}$ & $\begin{array}{c}0.088 \\
(0.008)\end{array}$ & $\begin{array}{c}0.285 \\
(0.679)\end{array}$ & & & \\
\hline France & $\begin{array}{c}0.054 \\
(0.029)\end{array}$ & $\begin{array}{c}0.111 \\
(0.006)\end{array}$ & $\begin{array}{c}0.492 \\
(0.260)\end{array}$ & & & \\
\hline Germany & $\begin{array}{c}0.038 \\
(0.031)\end{array}$ & $\begin{array}{c}0.121 \\
(0.009)\end{array}$ & $\begin{array}{c}0.313 \\
(0.259)\end{array}$ & & & \\
\hline Greece & $\begin{array}{c}0.014 \\
(0.074)\end{array}$ & $\begin{array}{c}0.111 \\
(0.017)\end{array}$ & $\begin{array}{c}0.132 \\
(0.671)\end{array}$ & & & \\
\hline Hong Kong & $\begin{array}{c}0.011 \\
(0.002)\end{array}$ & $\begin{array}{c}0.006 \\
(0.001)\end{array}$ & $\begin{array}{c}1.990 \\
(0.258)\end{array}$ & $\begin{array}{c}0.008 \\
(0.002)\end{array}$ & $\begin{array}{c}0.004 \\
(0.001)\end{array}$ & $\begin{array}{c}2.176 \\
(0.310)\end{array}$ \\
\hline Ireland & $\begin{array}{c}0.060 \\
(0.036)\end{array}$ & $\begin{array}{c}0.114 \\
(0.007)\end{array}$ & $\begin{array}{c}0.523 \\
(0.320)\end{array}$ & & & \\
\hline Italy & $\begin{array}{c}0.025 \\
(0.025)\end{array}$ & $\begin{array}{c}0.109 \\
(0.007)\end{array}$ & $\begin{array}{c}0.230 \\
(0.236)\end{array}$ & & & \\
\hline Japan & $\begin{array}{c}0.067 \\
(0.028)\end{array}$ & $\begin{array}{c}0.120 \\
(0.010)\end{array}$ & $\begin{array}{c}0.562 \\
(0.244)\end{array}$ & $\begin{array}{c}0.038 \\
(0.034)\end{array}$ & $\begin{array}{c}0.091 \\
(0.007)\end{array}$ & $\begin{array}{c}0.427 \\
(0.368)\end{array}$ \\
\hline Netherlands & $\begin{array}{c}0.102 \\
(0.031)\end{array}$ & $\begin{array}{c}0.108 \\
(0.008)\end{array}$ & $\begin{array}{c}0.951 \\
(0.297)\end{array}$ & & & \\
\hline New Zealand & $\begin{array}{c}0.094 \\
(0.048)\end{array}$ & $\begin{array}{c}0.099 \\
(0.006)\end{array}$ & $\begin{array}{c}0.943 \\
(0.510)\end{array}$ & $\begin{array}{c}0.150 \\
(0.044)\end{array}$ & $\begin{array}{c}0.098 \\
(0.009)\end{array}$ & $\begin{array}{c}1.539 \\
(0.551)\end{array}$ \\
\hline Norway & $\begin{array}{c}0.058 \\
(0.020)\end{array}$ & $\begin{array}{c}0.102 \\
(0.006)\end{array}$ & $\begin{array}{c}0.562 \\
(0.205)\end{array}$ & $\begin{array}{c}0.052 \\
(0.037)\end{array}$ & $\begin{array}{c}0.096 \\
(0.009)\end{array}$ & $\begin{array}{c}0.541 \\
(0.376)\end{array}$ \\
\hline Portugal & $\begin{array}{r}-0.013 \\
(0.037)\end{array}$ & $\begin{array}{c}0.116 \\
(0.009)\end{array}$ & $\begin{array}{r}-0.113 \\
(0.314)\end{array}$ & & & \\
\hline Singapore & $\begin{array}{c}0.011 \\
(0.014)\end{array}$ & $\begin{array}{c}0.044 \\
(0.003)\end{array}$ & $\begin{array}{c}0.249 \\
(0.307)\end{array}$ & $\begin{array}{c}0.011 \\
(0.016)\end{array}$ & $\begin{array}{c}0.044 \\
(0.004)\end{array}$ & $\begin{array}{c}0.236 \\
(0.340)\end{array}$ \\
\hline South Africa & $\begin{array}{c}0.017 \\
(0.054)\end{array}$ & $\begin{array}{c}0.156 \\
(0.015)\end{array}$ & $\begin{array}{c}0.111 \\
(0.347)\end{array}$ & $\begin{array}{c}0.026 \\
(0.065)\end{array}$ & $\begin{array}{c}0.160 \\
(0.016)\end{array}$ & $\begin{array}{c}0.165 \\
(0.399)\end{array}$ \\
\hline Spain & $\begin{array}{c}0.017 \\
(0.031)\end{array}$ & $\begin{array}{c}0.115 \\
(0.008)\end{array}$ & $\begin{array}{c}0.144 \\
(0.273)\end{array}$ & & & \\
\hline Sweden & $\begin{array}{c}0.055 \\
(0.024)\end{array}$ & $\begin{array}{c}0.108 \\
(0.008)\end{array}$ & $\begin{array}{c}0.505 \\
(0.240)\end{array}$ & $\begin{array}{c}0.084 \\
(0.034)\end{array}$ & $\begin{array}{c}0.097 \\
(0.006)\end{array}$ & $\begin{array}{c}0.864 \\
(0.350)\end{array}$ \\
\hline Switzerland & $\begin{array}{c}0.038 \\
(0.025)\end{array}$ & $\begin{array}{c}0.120 \\
(0.009)\end{array}$ & $\begin{array}{c}0.320 \\
(0.219)\end{array}$ & $\begin{array}{c}0.028 \\
(0.032)\end{array}$ & $\begin{array}{c}0.093 \\
(0.006)\end{array}$ & $\begin{array}{c}0.296 \\
(0.357)\end{array}$ \\
\hline U.K. & $\begin{array}{c}0.060 \\
(0.024)\end{array}$ & $\begin{array}{c}0.109 \\
(0.007)\end{array}$ & $\begin{array}{c}0.553 \\
(0.224)\end{array}$ & $\begin{array}{c}0.034 \\
(0.034)\end{array}$ & $\begin{array}{c}0.078 \\
(0.004)\end{array}$ & $\begin{array}{c}0.422 \\
(0.428)\end{array}$ \\
\hline Euro & $\begin{array}{c}0.084 \\
(0.030)\end{array}$ & $\begin{array}{c}0.085 \\
(0.007)\end{array}$ & $\begin{array}{c}0.979 \\
(0.362)\end{array}$ & $\begin{array}{c}0.084 \\
(0.030)\end{array}$ & $\begin{array}{c}0.085 \\
(0.007)\end{array}$ & $\begin{array}{c}0.979 \\
(0.362)\end{array}$ \\
\hline Equally weighted portfolio & $\begin{array}{c}0.054 \\
(0.013)\end{array}$ & $\begin{array}{c}0.065 \\
(0.006)\end{array}$ & $\begin{array}{c}0.828 \\
(0.209)\end{array}$ & $\begin{array}{c}0.053 \\
(0.017)\end{array}$ & $\begin{array}{c}0.043 \\
(0.003)\end{array}$ & $\begin{array}{c}1.233 \\
(0.393)\end{array}$ \\
\hline High-low portfolio & $\begin{array}{c}0.049 \\
(0.017)\end{array}$ & $\begin{array}{c}0.091 \\
(0.007)\end{array}$ & $\begin{array}{c}0.538 \\
(0.193)\end{array}$ & $\begin{array}{c}0.040 \\
(0.028)\end{array}$ & $\begin{array}{c}0.071 \\
(0.006)\end{array}$ & $\begin{array}{c}0.552 \\
(0.383) \\
\end{array}$ \\
\hline
\end{tabular}

Notes: Euro legacy currencies until 1998M12. Irish punt available from 1979M4. Japanese yen available from 1978M7. Austrailian dollar, Finnish markka, Greek drachma, Hong Kong dollar, New Zealand dollar, Singapore dollar, and South African rand available from 1997M1. All other currencies, the equally weighted portfolio, and the high-low portfolio are available for 1976M1-2007M6. Standard errors in parentheses. 
a) Equally Weighted and High-Low Portfolios (1979-2007)

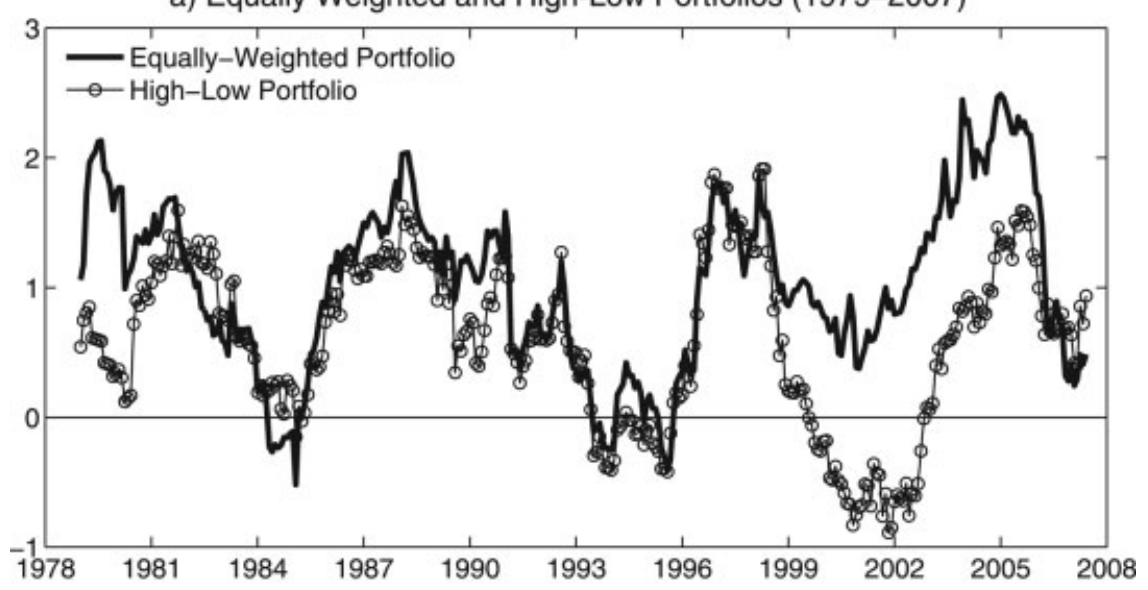

b) Equally Weighted Portfolio, New Zealand and Japan (1999-2007)

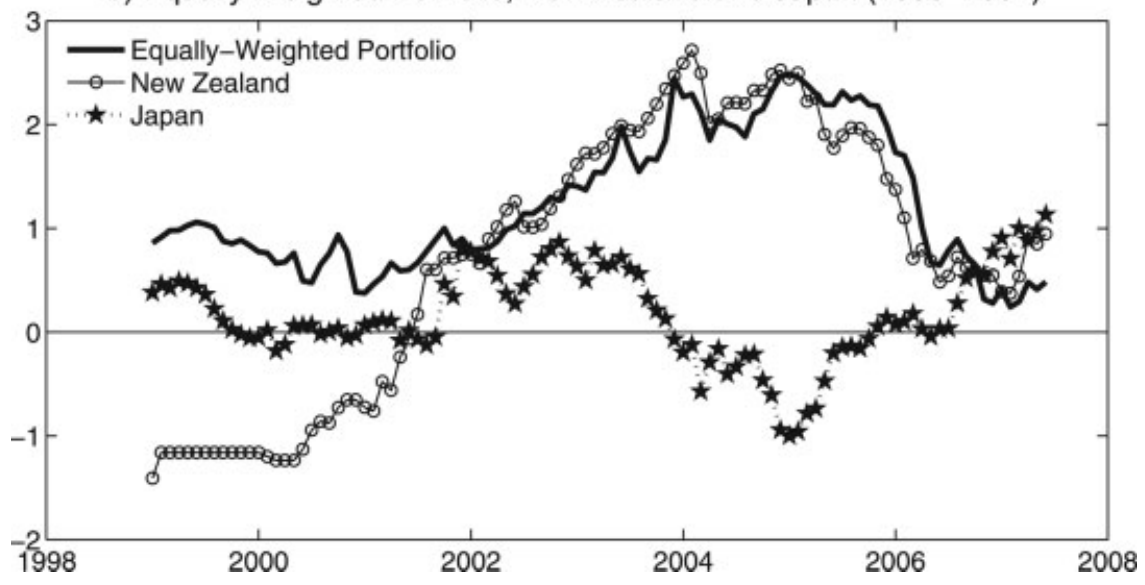

FIGURE 1. Realized Sharpe ratios of currency portfolios. Annualized, 36-month rolling window.

Panel a of Figure 1 displays a 36-month rolling window of the annualized Sharpe ratios associated with these strategies. Comparing the rolling Sharpe ratios we see that the one associated with the equally weighted carry trade is substantially less volatile than the one associated with the high-low strategy. Also note that there are very few periods in which the equally weighted carry trade has a negative rolling Sharpe ratio. Indeed, the rolling Sharpe ratio for the equally weighted carry-trade strategy has not been negative since the mid-1990s. Consistent with the attention that the carry trade has received in the financial press, the rolling Sharpe ratios for the equally weighted carry-trade strategy have been uniformly positive and large for most of the post-2000 period. In contrast, the rolling Sharpe ratio associated 
with the high-low strategy is consistently negative from the late 1990s to roughly 2003.

Relative to the currency-specific carry trade, the large rise in the Sharpe ratio associated with the equally weighted carry trade is due to a $52 \%$ fall in the standard deviation of the payoffs. Panel $b$ of Figure 1 illustrates this fact vividly for two currencies that have received much attention in the financial press as being widely used in carry-trade strategies. This panel displays, for the post-Euro period, the rolling Sharpe ratios associated with the equally weighted portfolios and those associated with the New Zealand and Japan carry-trade strategies. Notice that the rolling Sharpe ratios for New Zealand are uniformly negative from 1999 to 2001. Clearly the payoffs associated with the New Zealand carry trade are very volatile and were negative for an extended period of time. Table 1 indicates that, over the whole sample period, Japan's Sharpe ratio for Japan is large (0.16) and significantly different from zero. However, for the post-Euro period the Japan's Sharpe ratio is not statistically different from zero. This last result is illustrated by panel $b$ of Figure 1. The rolling Sharpe ratios associated with the Japan carry trade are not very large on average. Perhaps more significantly, they are consistently negative from 2003 to 2006.

Table 2 provides a different perspective on the gains from diversification. This table reports the skewness and kurtosis associated with the equally weighted, the high-low, and the currency-specific carry-trade strategies. For all three carry-trade strategies we can reject the hypothesis that the distribution of payoffs is normally distributed. But it is clear that, over the whole sample, the payoffs to the equally weighted carry trade are less skewed than the payoffs of the other strategies. This difference is particularly pronounced in the case of the high-low carry-trade strategy.

\section{Conclusion}

The carry-trade strategy is sometimes dismissed as involving "picking up pennies in front of a truck." This characterization seems appropriate for the currencyspecific and the high-low carry-trade strategies: The payoffs have low means and exhibit fat tails. However, this characterization is not appropriate for the equally weighted carry-trade strategy. In fact, the payoffs to this carry-trade strategy are less skewed than those of the value-weighted excess return to the U.S. stock market. The skewness of the excess returns to the value-weighted U.S. stock market is -0.78 for the whole sample and -0.72 for the post-Euro sample (see Table 3). ${ }^{3}$ In contrast, the skewness of the payoffs to the equally weighted carrytrade strategy is -0.17 both for the whole sample and the post-Euro sample.

3. We computed these estimates using data obtained from the Fama-French research database. 
TABLE 2. Higher moments of the excess returns to the carry-trade strategies.

\begin{tabular}{|c|c|c|c|c|c|c|}
\hline & \multicolumn{3}{|c|}{ 1976M1-2007M6 } & \multicolumn{3}{|c|}{ 1999M1-2007M6 } \\
\hline & Skewness & $\begin{array}{c}\text { Excess } \\
\text { Kurtosis }\end{array}$ & $\begin{array}{c}\text { Jarque-Bera } \\
\text { Statistic }\end{array}$ & Skewness & $\begin{array}{l}\text { Excess } \\
\text { Kurtosis }\end{array}$ & $\begin{array}{c}\text { Jarque-Bera } \\
\text { Statistic }\end{array}$ \\
\hline Australia & $\begin{array}{c}-0.363 \\
(0.235)\end{array}$ & $\begin{array}{c}-0.245 \\
(0.357)\end{array}$ & $\begin{array}{l}1.7 \\
(0.436)\end{array}$ & $\begin{array}{r}-0.366 \\
(0.239)\end{array}$ & $\begin{array}{c}-0.207 \\
(0.373)\end{array}$ & $\begin{array}{l}1.6 \\
(0.445)\end{array}$ \\
\hline Austria & $\begin{array}{r}-0.498 \\
(0.145)\end{array}$ & $\begin{array}{c}0.324 \\
(0.335)\end{array}$ & $\begin{array}{l}7.4 \\
(0.025)\end{array}$ & & & \\
\hline Belgium & $\begin{array}{r}-0.248 \\
(0.242)\end{array}$ & $\begin{array}{c}0.802 \\
(0.581)\end{array}$ & $\begin{array}{l}4.2 \\
(0.121)\end{array}$ & & & \\
\hline Canada & $\begin{array}{r}-0.574 \\
(0.179)\end{array}$ & $\begin{array}{c}1.086 \\
(0.516)\end{array}$ & $\begin{array}{l}25.8 \\
(0.000)\end{array}$ & $\begin{array}{r}-0.319 \\
(0.162)\end{array}$ & $\begin{array}{c}-0.529 \\
(0.207)\end{array}$ & $\begin{array}{l}2.1 \\
(0.352)\end{array}$ \\
\hline Denmark & $\begin{array}{c}-0.179 \\
(0.189)\end{array}$ & $\begin{array}{c}1.405 \\
(0.514)\end{array}$ & $\begin{array}{l}25.1 \\
(0.000)\end{array}$ & $\begin{array}{c}-0.157 \\
(0.341)\end{array}$ & $\begin{array}{c}0.460 \\
(0.535)\end{array}$ & $\begin{array}{l}1.2 \\
(0.545)\end{array}$ \\
\hline Finland & $\begin{array}{c}0.125 \\
(0.258)\end{array}$ & $\begin{array}{r}-0.330 \\
(0.492)\end{array}$ & $\begin{array}{l}0.2 \\
(0.921)\end{array}$ & & & \\
\hline France & $\begin{array}{c}0.013 \\
(0.200)\end{array}$ & $\begin{array}{c}0.774 \\
(0.377)\end{array}$ & $\begin{array}{l}4.9 \\
(0.085)\end{array}$ & & & \\
\hline Germany & $\begin{array}{r}-0.294 \\
(0.154)\end{array}$ & $\begin{array}{c}0.725 \\
(0.306)\end{array}$ & $\begin{array}{c}6.0 \\
(0.050)\end{array}$ & & & \\
\hline Greece & $\begin{array}{c}-0.872 \\
(0.422)\end{array}$ & $\begin{array}{c}1.535 \\
(0.475)\end{array}$ & $\begin{array}{l}5.2 \\
(0.075)\end{array}$ & & & \\
\hline Hong Kong & $\begin{array}{c}3.294 \\
(0.721)\end{array}$ & $\begin{array}{l}16.130 \\
(5.535)\end{array}$ & $\begin{array}{l}>1000 \\
(0.000)\end{array}$ & $\begin{array}{c}2.078 \\
(0.442)\end{array}$ & $\begin{array}{c}6.442 \\
(2.633)\end{array}$ & $\begin{array}{l}188.6 \\
(0.000)\end{array}$ \\
\hline Ireland & $\begin{array}{c}-0.130 \\
(0.244)\end{array}$ & $\begin{array}{c}0.591 \\
(0.454)\end{array}$ & $\begin{array}{l}2.5 \\
(0.285)\end{array}$ & & & \\
\hline Italy & $\begin{array}{c}-0.349 \\
(0.225)\end{array}$ & $\begin{array}{c}1.108 \\
(0.518)\end{array}$ & $\begin{array}{l}18.3 \\
(0.000)\end{array}$ & & & \\
\hline Japan & $\begin{array}{c}-0.962 \\
(0.382)\end{array}$ & $\begin{array}{c}3.217 \\
(1.205)\end{array}$ & $\begin{array}{l}125.9 \\
(0.000)\end{array}$ & $\begin{array}{r}-0.088 \\
(0.180)\end{array}$ & $\begin{array}{c}-0.077 \\
(0.234)\end{array}$ & $\begin{array}{l}0.2 \\
(0.925)\end{array}$ \\
\hline Netherlands & $\begin{array}{c}-0.261 \\
(0.200)\end{array}$ & $\begin{array}{c}0.589 \\
(0.364)\end{array}$ & $\begin{array}{l}3.0 \\
(0.221)\end{array}$ & & & \\
\hline New Zealand & $\begin{array}{r}-0.316 \\
(0.223)\end{array}$ & $\begin{array}{c}0.079 \\
(0.283)\end{array}$ & $\begin{array}{l}1.5 \\
(0.479)\end{array}$ & $\begin{array}{r}-0.530 \\
(0.242)\end{array}$ & $\begin{array}{c}0.476 \\
(0.360)\end{array}$ & $\begin{array}{l}4.0 \\
(0.136)\end{array}$ \\
\hline Norway & $\begin{array}{r}-0.294 \\
(0.208)\end{array}$ & $\begin{array}{c}1.153 \\
(0.482)\end{array}$ & $\begin{array}{l}19.6 \\
(0.000)\end{array}$ & $\begin{array}{r}-0.039 \\
(0.218)\end{array}$ & $\begin{array}{c}0.401 \\
(0.307)\end{array}$ & $\begin{array}{l}0.5 \\
(0.762)\end{array}$ \\
\hline Portugal & $\begin{array}{c}-0.933 \\
(0.333)\end{array}$ & $\begin{array}{c}3.035 \\
(1.025)\end{array}$ & $\begin{array}{l}80.4 \\
(0.000)\end{array}$ & & & \\
\hline Singapore & $\begin{array}{c}0.083 \\
(0.183)\end{array}$ & $\begin{array}{c}0.200 \\
(0.427)\end{array}$ & $\begin{array}{l}0.3 \\
(0.877)\end{array}$ & $\begin{array}{c}0.019 \\
(0.186)\end{array}$ & $\begin{array}{c}0.321 \\
(0.474)\end{array}$ & $\begin{array}{l}0.4 \\
(0.833)\end{array}$ \\
\hline South Africa & $\begin{array}{c}-0.274 \\
(0.181)\end{array}$ & $\begin{array}{c}0.451 \\
(0.532)\end{array}$ & $\begin{array}{l}2.6 \\
(0.275)\end{array}$ & $\begin{array}{r}-0.314 \\
(0.182)\end{array}$ & $\begin{array}{c}0.051 \\
(0.452)\end{array}$ & $\begin{array}{l}1.7 \\
(0.437)\end{array}$ \\
\hline Spain & $\begin{array}{c}-0.931 \\
(0.386)\end{array}$ & $\begin{array}{c}2.619 \\
(1.687)\end{array}$ & $\begin{array}{l}98.1 \\
(0.000)\end{array}$ & & & \\
\hline Sweden & $\begin{array}{r}-0.776 \\
(0.439)\end{array}$ & $\begin{array}{c}3.952 \\
(1.405)\end{array}$ & $\begin{array}{l}209.6 \\
(0.000)\end{array}$ & $\begin{array}{c}-0.080 \\
(0.256)\end{array}$ & $\begin{array}{c}-0.085 \\
(0.241)\end{array}$ & $\begin{array}{l}0.1 \\
(0.942)\end{array}$ \\
\hline Switzerland & $\begin{array}{c}-0.173 \\
(0.288)\end{array}$ & $\begin{array}{c}1.062 \\
(0.548)\end{array}$ & $\begin{array}{l}11.6 \\
(0.003)\end{array}$ & $\begin{array}{r}-0.612 \\
(0.158)\end{array}$ & $\begin{array}{c}-0.280 \\
(0.315)\end{array}$ & $\begin{array}{l}5.7 \\
(0.059)\end{array}$ \\
\hline U.K. & $\begin{array}{c}-0.023 \\
(0.405)\end{array}$ & $\begin{array}{c}1.938 \\
(0.966)\end{array}$ & $\begin{array}{l}44.8 \\
(0.000)\end{array}$ & $\begin{array}{r}-0.120 \\
(0.196)\end{array}$ & $\begin{array}{c}-0.485 \\
(0.215)\end{array}$ & $\begin{array}{l}0.9 \\
(0.645)\end{array}$ \\
\hline Euro & $\begin{array}{c}-0.169 \\
(0.358)\end{array}$ & $\begin{array}{c}0.576 \\
(0.694)\end{array}$ & $\begin{array}{l}1.8 \\
(0.417)\end{array}$ & $\begin{array}{r}-0.169 \\
(0.358)\end{array}$ & $\begin{array}{c}0.576 \\
(0.694)\end{array}$ & $\begin{array}{l}1.8 \\
(0.417)\end{array}$ \\
\hline Equally weighted portfolio & $\begin{array}{r}-0.178 \\
(0.437)\end{array}$ & $\begin{array}{c}4.452 \\
(1.176)\end{array}$ & $\begin{array}{l}313.3 \\
(0.000)\end{array}$ & $\begin{array}{r}-0.166 \\
(0.232)\end{array}$ & $\begin{array}{c}0.533 \\
(0.454)\end{array}$ & $\begin{array}{l}1.7 \\
(0.433)\end{array}$ \\
\hline High-low Portfolio & $\begin{array}{c}-0.720 \\
(0.286)\end{array}$ & $\begin{array}{c}3.054 \\
(0.753)\end{array}$ & $\begin{array}{l}179.1 \\
(0.000)\end{array}$ & $\begin{array}{r}-0.340 \\
(0.143)\end{array}$ & $\begin{array}{c}-0.005 \\
(0.403)\end{array}$ & $\begin{array}{l}2.0 \\
(0.374)\end{array}$ \\
\hline
\end{tabular}

Note: Euro legacy currencies until 1998M12. Irish punt available from 1979M4. Japanese yen available from 1978M7. Austrailian dollar, Finnish markka, Greek drachma, Hong Kong dollar, New Zealand dollar, Singapore dollar, and South African rand available from 1997M1. All other currencies, the equally weighted portfolio, and the high-low portfolio are available for 1976M1-2007M6. Standard errors in parentheses for skewness and kurtosis, $p$-value for the Jarque-Bera statistic. 
TABLE 3. Excess returns to the value-weighted U.S. stock market (statistics presented on an annualized basis).

\begin{tabular}{|c|c|c|c|c|c|}
\hline \multicolumn{3}{|c|}{ 1976M1-2007M6 } & \multicolumn{3}{|c|}{ 1999M1-2007M6 } \\
\hline Mean & $\begin{array}{c}\text { Standard } \\
\text { Deviation }\end{array}$ & Sharpe Ratio & Mean & $\begin{array}{c}\text { Standard } \\
\text { Deviation }\end{array}$ & Sharpe Ratio \\
\hline $\begin{array}{l}0.073 \\
(0.025)\end{array}$ & $\begin{array}{c}0.149 \\
(0.009)\end{array}$ & $\begin{array}{c}0.488 \\
(0.184)\end{array}$ & $\begin{array}{c}0.065 \\
(0.047)\end{array}$ & $\begin{array}{c}0.156 \\
(0.015)\end{array}$ & $\begin{array}{c}0.418 \\
(0.322)\end{array}$ \\
\hline \multicolumn{6}{|c|}{ Higher moments of the excess return to the value-weighted U.S. stock Market. } \\
\hline \multicolumn{3}{|c|}{ 1976M1-2007M6 } & \multicolumn{3}{|c|}{ 1999M1-2007M6 } \\
\hline Skewness & $\begin{array}{l}\text { Excess } \\
\text { Kurtosis }\end{array}$ & $\begin{array}{c}\text { Jarque-Bera } \\
\text { Statistic }\end{array}$ & Skewness & $\begin{array}{c}\text { Excess } \\
\text { Kurtosis }\end{array}$ & $\begin{array}{c}\text { Jarque-Bera } \\
\text { Statistic }\end{array}$ \\
\hline $\begin{array}{l}-0.777 \\
(0.350)\end{array}$ & $\begin{array}{c}2.738 \\
(1.570)\end{array}$ & $\begin{array}{l}155.7 \\
(0.000)\end{array}$ & $\begin{array}{c}-0.724 \\
(0.249)\end{array}$ & $\begin{array}{c}0.737 \\
(0.909)\end{array}$ & $\begin{array}{l}13.8 \\
(0.001)\end{array}$ \\
\hline
\end{tabular}

Notes: Standard errors in parentheses, except for the Jarque-Bera statistic, for which the $p$-value is shown.

It is always possible that the positive returns to the carry trade reflect a peso problem. Indeed, it is almost surely the case that the high Sharpe ratio associated with the Hong-Kong dollar carry trade (2.18 for the post-Euro sample) reflects political risk associated with mainland China that, to date, has not materialized. It is much less obvious what particular peso problem can explain the high Sharpe ratio associated with the equally weighted carry trade. That strategy seems to involve "picking up pennies in front of an unknown truck that has never been seen."

\section{References}

Bilson, John F. O. (1981). “The 'Speculative Efficiency' Hypothesis.” Journal of Business, 54, 435-451.

Burnside, Craig, Martin Eichenbaum, Isaac Kleshchelski, and Sergio Rebelo (2006). "The Returns to Currency Speculation.” National Bureau of Economic Research Working Paper No. 12489.

Engel, Charles (1996). "The Forward Discount Anomaly and the Risk Premium: A Survey of Recent Evidence." Journal of Empirical Finance 3, 123-192.

Fama, Eugene F. (1984). "Forward and Spot Exchange Rates.” Journal of Monetary Economics, $14,319-338$.

Hodrick, Robert J. (1987). The Empirical Evidence on the Efficiency of Forward and Futures Foreign Exchange Markets. Harwood Academic. 